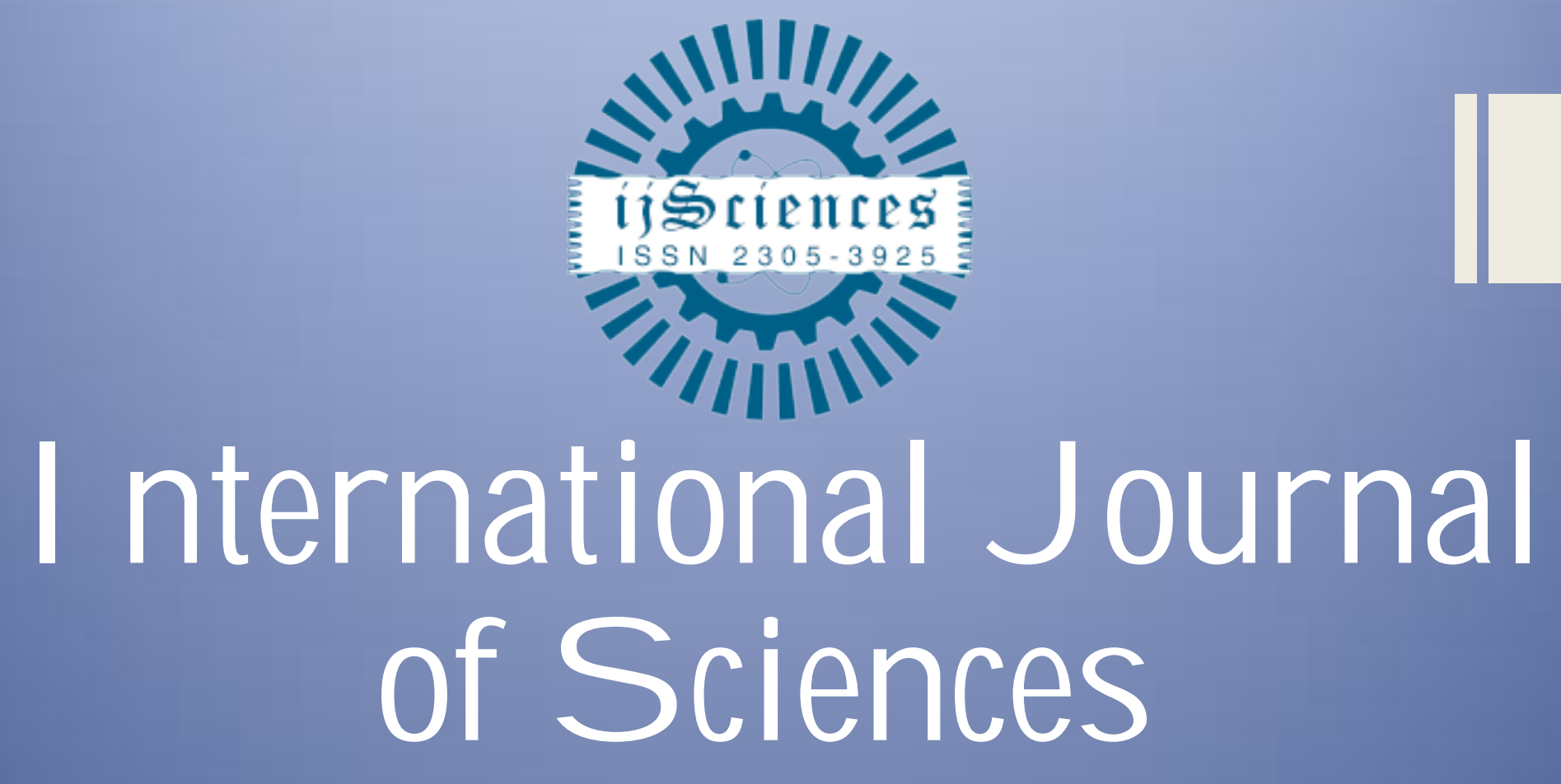

Volume 4 - January 2015

\title{
Alkhaer Publications
}

ijSciences [ ISSN 2305-3925 ] is an open access journal having expertise in delivering high-quality and rapid publications, from online submission systems and in-depth peer review to an efficient, author-friendly production process.

Web: ww. ijsciences.com 


\title{
Periodic Distortions in Nematics caused by Flexoelectricity and Saddle-Splay Elasticity
}

\section{Amelia Carolina Sparavigna ${ }^{1}$}

${ }^{1}$ Department of Applied Science and Technology, Politecnico di Torino, Italy

\begin{abstract}
In liquid crystal materials, the coupling between their elastic field and an external action, such as electric and magnetic fields or the confinement created by free surfaces or cell walls, can give rise to periodic domains. Here, some simple calculations are proposed for nematics in planar cells, where undulations are caused by flexoelectricity and saddle-splay elasticity.
\end{abstract}

Keywords: Liquid Crystals; Nematics; Flexoelectricity; Saddle-Splay Elasticity

\section{Introduction}

Periodic domains are well-known in cholesteric or ferroelectric liquid crystals, because these materials possess a natural periodic helicoidal distribution of the molecular order [1-3]. Periodic modulations can appear in nematic liquid crystals too, controlled by external factors such as applied fields, cell thicknesses and asymmetric conditions of anchoring at the cell walls [4,5]. An electric field, for instance, is controlling the instability produced by the flexoelectric effect.

Periodic domains caused by flexoelectricity were first observed by Vistin and theoretically studied by Bobylev, Chigrinov and Pikin [6,7]. More recently, Lavrentovich and Pergamenshchick discovered the periodic instability controlled by the saddle-splay elastic contribution to the free energy density [8].

Here we are discussing, with a simple and intuitive approach, the behaviours of nematics when flexoelectricity or saddle-splay elasticity are present. We will see how the free energy can be described and how it is producing periodic textures. The presence and value of thresholds for field and cell thickness are also discussed.

\section{Director field and its derivatives}

A fundamental vector field in nematic liquid crystals is the director $\vec{n}$, describing the local orientation, in average, of molecules. This is usually a unit vector. Using it we can give the free energy density of a nematic cell as:

$f=\frac{K}{2}\left[(\operatorname{div} \vec{n})^{2}+(\vec{n} \cdot \operatorname{rot} \vec{n})^{2}+(\vec{n} \times \operatorname{rot} \vec{n})^{2}\right]$

Eq.1 is given in the case of the uniform elastic approximation. If the nematic liquid crystal is subjected to an electric field, we have to add another term to (1), $f_{E}=-\varepsilon_{o} \vec{D} \cdot \vec{E} / 2$, usually given as:

$$
f_{E}=-\frac{\varepsilon_{o} \Delta \varepsilon}{2}(\vec{E} \cdot \vec{n})^{2}
$$

Analogous term is given in the case of the presence of a magnetic field. The term in (2) has the sign opposite to the well-known density of energy of an electric field in a dielectric; in fact, (2) is obtained by considering fixed the difference of potential of the cell containing the liquid crystal [9].

In (2), it is appearing the dielectric anisotropy $\Delta \varepsilon$ of nematic [10]. It is $\Delta \varepsilon=\varepsilon_{\|}-\varepsilon_{\perp}$, where $\varepsilon_{\|}, \varepsilon_{\perp}$ are the dielectric constants, parallel and perpendicular to the director. The displacement vector is given by $\vec{D}=\varepsilon \vec{E}$, where $\varepsilon$ is a tensor.

In the nematic liquid crystal we have a privileged direction, that of director $\vec{n}$, so we can consider the electric field in the components parallel and perpendicular to the director, that is $\vec{E}=\vec{E}_{\|}+\vec{E}_{\perp}$. We have, in the uniaxial nematic phase, $\vec{D}=\varepsilon_{\|} \vec{E}_{\|}+\varepsilon_{\perp} \vec{E}_{\perp}$. Since $\vec{E}_{\|}=(\vec{n} \cdot \vec{E}) \vec{n}$, we can write the displacement vector in the following manner: $\vec{D}=\varepsilon_{\perp} \vec{E}_{\perp}+\varepsilon_{\|} \vec{E}_{\|}=\varepsilon_{\perp} \vec{E}_{\perp}+\varepsilon_{\perp} \vec{E}_{\|}$ $-\varepsilon_{\perp} \vec{E}_{\|}+\varepsilon_{\|} \vec{E}_{\|}=\varepsilon_{\perp} \vec{E}+\left(\varepsilon_{\|}-\varepsilon_{\perp}\right)(\vec{n} \cdot \vec{E}) \vec{n}$.

Then:

$\vec{D}=\varepsilon_{\perp} \vec{E}+\left(\varepsilon_{\|}-\varepsilon_{\perp}\right)(\vec{n} \cdot \vec{E}) \vec{n}$

So we have: 


$$
\begin{gathered}
f_{E}=-\frac{\varepsilon_{o}}{2} \vec{D} \cdot \vec{E} \\
=-\frac{\varepsilon_{O}}{2}\left[\varepsilon_{\perp} \vec{E}+\left(\varepsilon_{\|}-\varepsilon_{\perp}\right)(\vec{n} \cdot \vec{E}) \vec{n}\right] \cdot \vec{E} \\
=-\frac{\varepsilon_{o}}{2} \varepsilon_{\perp} E^{2}-\frac{\varepsilon_{o}}{2} \Delta \varepsilon(\vec{n} \cdot \vec{E})^{2}
\end{gathered}
$$

The first term does not depend on the director field. For this reason, it is not considered in the distortional contribution to the bulk free energy density.

Besides director, there is another vector, obtained from its derivatives, which can be used. Let us define it as vector $\vec{A}=\vec{A}_{1}+\vec{A}_{3}$ :

$$
\vec{A}=\vec{n}(\vec{\nabla} \cdot \vec{n})-(\vec{n} \cdot \vec{\nabla}) \vec{n}
$$

$$
=\vec{n} \operatorname{div} \vec{n}+\vec{n} \times \operatorname{rot} \vec{n}=\vec{A}_{1}+\vec{A}_{3}
$$

$\vec{A}$ is the sum of two vectors: one has the magnitude given by the divergence of the director, the other is the cross product of the director with its rotor. We can find $\vec{A}_{1}, \vec{A}_{3}$ in the contribution to the bulk free energy density of flexoelectricity: $f_{\text {Flexo }}=-\vec{P} \cdot \vec{E}$.

Flexoelectricity is a property of liquid crystals, similar to the piezoelectric effect. In certain anisotropic materials, which contain molecular asymmetry or quadrupolar ordering with permanent molecular dipoles, an applied electric field may induce a distortion of the director orientation. Conversely, any distortion induces a macroscopic polarization within the material. The polarization vector $\vec{P}$ in the flexoelectric term can be described as:

$$
\begin{gathered}
\vec{P}=e_{S} \vec{n}(\vec{\nabla} \cdot \vec{n})-e_{B}(\vec{n} \cdot \vec{\nabla}) \vec{n}=e_{S} \vec{n} \operatorname{div} \vec{n} \\
+e_{B} \vec{n} \times \operatorname{rot} \vec{n}=e_{S} \vec{A}_{1}+e_{B} \vec{A}_{3}
\end{gathered}
$$

In (6), we have used vectors $\vec{A}_{1}, \vec{A}_{3}$, which can be defined as distortional Lifshitz vectors $[11,12]$; in [11] we have discussed the role of these vectors and helicity in the nematic free energy density.

The coupling of polarization $\vec{P}$ with an external electric field results in the appearance of a periodic distortion from an initial planar orientation of the nematic cell [7]. Meyer showed that the infinite liquid crystal must be disturbed, the perturbation being periodic along the director orientation and the period is inversely proportional to electric field strength [13].

Flexoelectricity in liquid crystals is analogous with piezoelectricity in solids. In the piezoelectric materials, an applied uniform strain can induce an electric polarization and vice versa. Some crystallographic considerations restrict this property to non-centrosymmetric systems.

\section{Periodic distortions in nematics}

Let us discuss the results from $[7,13]$, that is the periodic distortion in the infinite medium caused by flexoelectricity. The free energy density is given by:

$$
\begin{aligned}
& f=\frac{K}{2}\left[(\operatorname{div} \vec{n})^{2}+(\vec{n} \cdot \operatorname{rot} \vec{n})^{2}+(\vec{n} \times \operatorname{rot} \vec{n})^{2}\right] \\
& -e \vec{E} \cdot(\vec{n} \operatorname{div} \vec{n}+\vec{n} \times \operatorname{rot} \vec{n})
\end{aligned}
$$

in the uniform elastic approximation with $K$ elastic constant, and with the dielectric anisotropy negligible. Moreover we assumed $e_{B} \approx e_{S} \approx e$. In the case of different flexoelectric coefficients:

$$
\begin{aligned}
& f=\frac{K}{2}\left[(\operatorname{div} \vec{n})^{2}+(\vec{n} \cdot \operatorname{rot} \vec{n})^{2}+(\vec{n} \times \operatorname{rot} \vec{n})^{2}\right] \\
& -e_{S} \vec{E} \cdot \vec{A}_{1}-e_{B} \vec{A}_{3}
\end{aligned}
$$

Let us consider the director $\vec{n}$ in a uniform configuration, as a vector parallel to x-axis and the electric field $\vec{E}$ parallel to z-axis as $\vec{E}=E \vec{k}$, where $\vec{k}$ is the unit vector of z-axis. Angles $\theta$ and $\phi$ are shown in the Figure 1.

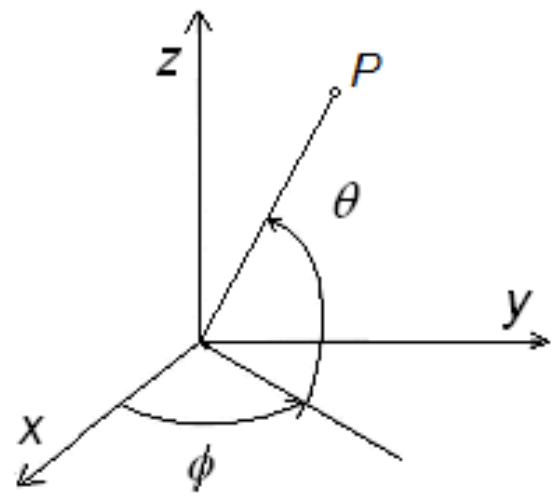

Figure 1: Frame of reference and angles used for calculations.

The components of director $\vec{n}$ are $n_{x}=\cos \theta$, $n_{y}=0, n_{z}=\sin \theta$, when we have $\phi=0$.

Let us consider a deformation of $\vec{n}$ depending on $\mathrm{x}$. In the case of an infinite nematic medium without deformations of the director, the free energy density is zero. If we had a tilt angle variation of the form $\theta=-e x E / K$, we should 
have a periodic deformation of director $\vec{n}$, with a period inversely proportional to the electric field strength.

The free energy density of the distorted configuration, including the flexoelectric term, is:

$$
\begin{aligned}
f_{\text {dist }} & =\frac{1}{2} K\left(\frac{\partial \theta}{\partial x}\right)^{2}+e E \frac{\partial \theta}{\partial x} \\
& =-\frac{1}{2} e \frac{E^{2}}{K}<0
\end{aligned}
$$

Then, a periodic distortion in a non-confined nematic is possible because it has a free energy density lower than that possessed by the uniform configuration. Let us note that there is no threshold for the electric field. Even a small field gives rise to the distorted configuration.

The existence of a threshold is a consequence of the medium confinement. Let us imagine a nematic material confined in a cell composed by two plane walls, both parallel to $[x, y]$ plane, at a distance $d$. The anchoring conditions must be included in the energy balance. We can assume a surface energy density of the Rapini-Papoular form $f=-W \cos ^{2} \theta$, for a surface treatment favouring a molecular alignment parallel to $\mathrm{x}$-axis. If the director field $\vec{n}$ is uniform in the planar alignment, $f=-W$.

Let us assume, as in Ref. [7], the behaviour of the tilt angle in the form $\theta=-\alpha x E / K$, with $\alpha$ being dimensioned as charge/length. We can integrate the free energy density on a volume $V$ given by $V=d \Lambda L$, where $d$ is the cell thickness, $L$ a fixed length in y-direction and $\Lambda$ the director distortion wavelength along the x-direction, we obtain:

$$
F_{\text {dist }}=\frac{E^{2} d \Lambda L}{K}\left[\frac{1}{2} \alpha^{2}-e \alpha\right]-\frac{1}{2} W \Lambda L
$$

The last term in (10) is the surface energy contribution. In the case of a uniform director field, we have a total energy as $F_{\text {unif }}=-W \Lambda L$. The behaviour of the two free energies $F_{\text {dist }}, F_{\text {unif }}$ is given in Figure 2: we can see the existence of a threshold field $E *$.

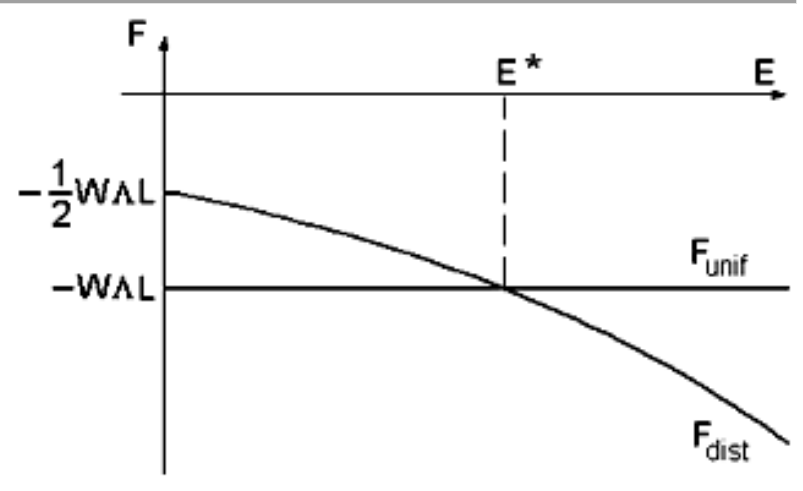

Figure 2: Free energy as a function of the electric field for the uniform configuration and the distorted one.

If the electric field has a value $E<E^{*}$, the stable configuration of the director field is that with lower energy: in this case, it means when the director field is uniform. When $E>E *$, the stable configuration is the distorted one. Comparing the two values of the total energy, that is:

$$
F_{\text {dist }} \approx F_{\text {unif }}
$$

we can approximately find the threshold electric field as:

$(E *)^{2}\left(\frac{1}{2} \alpha^{2} d-e \alpha d\right)=-\frac{W}{2} K$,

where $\left(\alpha^{2} d / 2-e \alpha d\right)<0$, to have a real electric field:

$\left(E^{*}\right)^{2}=\frac{W}{d} \frac{K}{\left|\alpha^{2}-2 e \alpha\right|}$.

The threshold field has a value:

$E^{*}=\left(\frac{W K}{\alpha d|\alpha-2 e|}\right)^{1 / 2}=\left(\frac{W K}{a^{2} d}\right)^{1 / 2}$

where $\alpha<2 e$. If $a^{2}=|\alpha(\alpha-2 e)| \approx e^{2}$, and assuming the parameters as $W=10^{-4} \mathrm{~J} / \mathrm{m}^{2}, K=$ $10^{-11} \mathrm{~N}, d=10 \mu \mathrm{m}$, we have $a=10^{-11} \mathrm{C} / \mathrm{m}$ and we find a threshold voltage of $\approx 10$ Volt .

\section{Flexoelectricity and hybrid cell}

Let us consider the role of flexoelectricity in a hybrid nematic cell. This is a cell where the nematic is confined between two parallel walls with different anchoring conditions. One surface is treated to favour planar 
alignment; the other is favouring homeotropic alignment. The cell is then known as HAN, Hybrid Aligned Nematic, cell. The hybrid cell we discuss has the $\mathrm{z}$-axis perpendicular to cell walls ( Figure 3 ).

An electric field can be applied parallel to z-axis: we have then $\vec{E}=E \vec{k}$ where $\overrightarrow{\mathrm{k}}$ is the unit vector of z-axis. $\overrightarrow{\mathrm{k}}$ is the homeotropic direction too. The unit vector $\vec{i}$, parallel to the cell walls, gives the easy planar direction. The bulk free energy density is given, in the elastic isotropic approximation, by:

$$
f=\frac{K}{2}\left[(\operatorname{div} \vec{n})^{2}+(\operatorname{rot} \vec{n})^{2}\right]-\frac{\varepsilon_{o} \Delta \varepsilon}{2}(\vec{E} \cdot \vec{n})^{2}
$$

where the last term is due to the dielectric anisotropy $\Delta \varepsilon$ of the nematic.

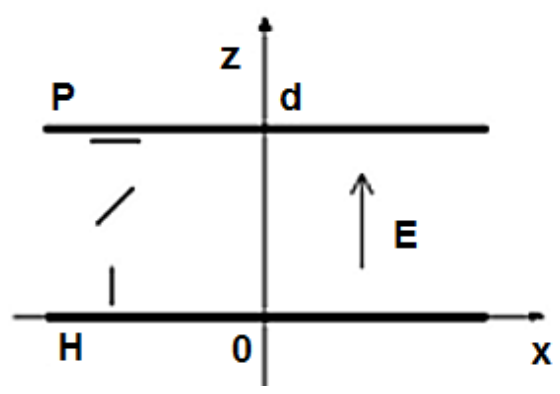

Figure 3: Frame of reference for the hybrid cell.

The surface energy density in the Rapini-Papoular form can be used:

$$
\begin{aligned}
& f_{\text {Surf }}=W_{P}(\vec{n} \cdot \vec{i})^{2} \\
& f_{\text {Surf }}=-W_{H}(\vec{n} \cdot \vec{k})^{2}
\end{aligned}
$$

at the two surfaces, for $\mathrm{Z}=\mathrm{d}$ and for $\mathrm{Z}=0$. $W_{P}, W_{H}$ are energy densities of the surface anchoring $\left(W_{P}, W_{H}>0\right)$.

If we have a planar cell with surface $S$, thickness $d$, and a uniform director configuration $\vec{n}=n \vec{i}$, the total free energy is $F_{P}=-2 S W_{P}$. If the director configuration is a uniform homeotropic one, then $\vec{n}=n \vec{k}$, the total free energy is the sum of the energy due to the presence of electric field and surfaces: $F_{H}=-\varepsilon_{o} \Delta \varepsilon E^{2} / 2$ $-2 S W_{H}$.

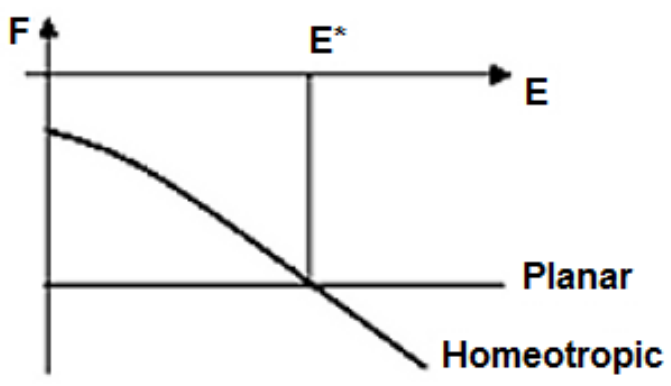

Figure 4: Free energies as a function of electric field, in the case of planar and homeotropic configurations. Note the presence of a threshold.

Graphically comparing (Figure 4) the energies of homeotropic and planar cells, in the case $W_{P}>W_{H}$, we see the possibility of an electric threshold field $E^{*}$ : under this value of the electric field, it is favoured the planar configuration, over the threshold value, it is the homeotropic configuration that has a lower energy.

In a hybrid cell, the director changes from a planar configuration at one of the cell wall, to a homeotropic configuration at the other cell wall. The tilt angle is then depending on $\mathrm{z}$, as a function $\theta=\theta(z)$. The director field is given by: $\vec{n}=\cos \theta \vec{i}+\sin \theta \vec{k}$.

If the anchoring is strong, the tilt angle is $\theta=\pi / 2$ at $z=0$, the homeotropic wall, and $\theta=0$ at $z=d$, the planar wall. In the one elastic constant approximation, we have the bulk free energy density in the form:

$$
f=\frac{K}{2}\left(\frac{\partial \theta}{\partial z}\right)^{2}-\frac{\varepsilon_{o} \Delta \varepsilon}{2} E^{2} \sin ^{2} \theta
$$

and the surface energy density $f_{\text {Surf }}=-W_{P}-W_{H}$. Let us represent approximately the hybrid configuration with a linear function of the tilt angle:

$\theta=-\frac{\pi}{2} \frac{z}{d}+\frac{\pi}{2}$

with $\theta_{o}=\pi / 2$ and $\theta_{d}=0$.

Then $z=-\pi / 2 d$ and the total bulk energy is:

$$
\begin{aligned}
& F=S \int_{0}^{d} \frac{K}{2}\left(\frac{\partial \theta}{\partial z}\right)^{2} d z-\frac{E^{2} \varepsilon_{o} \Delta \varepsilon}{2} \\
& \times S \int_{0}^{d} \sin ^{2} \theta d z=\frac{K \pi^{2} S}{8 d}-\frac{E^{2} \varepsilon_{o} \Delta \varepsilon}{4} S d
\end{aligned}
$$




$$
F=\frac{K \pi^{2} S}{8 d}-\frac{\varepsilon_{o} \Delta \varepsilon E^{2}}{4} S d-S\left(W_{P}-W_{H}\right)
$$

Let us compare this expression with the energy of the cell in homeotropic and planar configurations, choosing an anchoring energy favouring planar and hybrid configurations under threshold fields:

$$
\begin{gathered}
F_{P}=-2 S W_{P} \\
F_{H}=-\frac{\varepsilon_{o} \Delta \varepsilon}{2} E^{2} S d-2 S W_{H} \\
F_{H y b} \\
=\frac{K \pi^{2} S}{8 d}-\frac{\varepsilon_{o} \Delta \varepsilon}{4} E^{2} S d-S\left(W_{P}-W_{H}\right)
\end{gathered}
$$

The free energies are shown in Figures 5, in the case of suitable anchoring parameters $\left(W_{P}>W_{H}\right)$.

We observe two threshold fields: when the field is lower than $E^{\prime}$, the nematic is planar, if the field is comprised between $E^{\prime}$ and $E^{\prime \prime}$, the cell is hybrid. Above the second threshold $E^{\prime \prime}$, the cell is homeotropic.

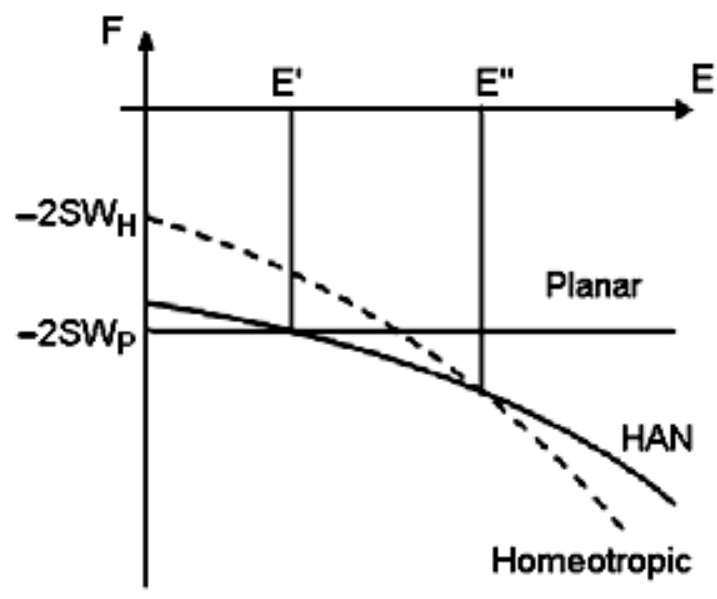

Figure 5: Free energy of planar, hybrid (HAN) and homeotropic configurations, as function of the electric field. Note the existence of two thresholds. One is for the transition between planar and HAN configuration, the other between HAN and homeotropic configuration.

As previously discussed, the electric field can be coupled with a polarization coming from an elastic deformation in the flexoelectric effect. In planar and homeotropic configurations, the director is uniform and therefore the flexoelectric effect is absent. In the hybrid cell, the deformation exists and gives a flexoelectric polarization
$\overrightarrow{\mathrm{P}}=e_{S} \overrightarrow{\mathrm{n}} \operatorname{div} \overrightarrow{\mathrm{n}}+e_{B} \overrightarrow{\mathrm{n}} \times \operatorname{rot} \overrightarrow{\mathrm{n}}$. Let us add the term $f_{\text {Flexo }}=-\vec{P} \cdot \vec{E}$ to the free energy density:

$$
\begin{aligned}
& f_{\text {Flexo }}=-\vec{P} \cdot \vec{E} \\
& =-\left(e_{S}-e_{B}\right) E \sin \theta \cos \theta \frac{\partial \theta}{\partial z}
\end{aligned}
$$

If $\theta$ is given by (18), after integrating on the cell volume, we have the contribution of flexoelectricity to the total free energy as:

$$
F_{\text {flexo }}=-\left(e_{S}-e_{B}\right) E S
$$

In principle, the coefficient $\left(e_{S}-e_{B}\right)$ could be positive or negative, depending on the value of splay and bend parameters. The threshold values $E^{\prime}, E^{\prime \prime}$ are changed from the contribution of the flexoelectricity. They could be lowered or raised by the induced polarization (see Figure 6).

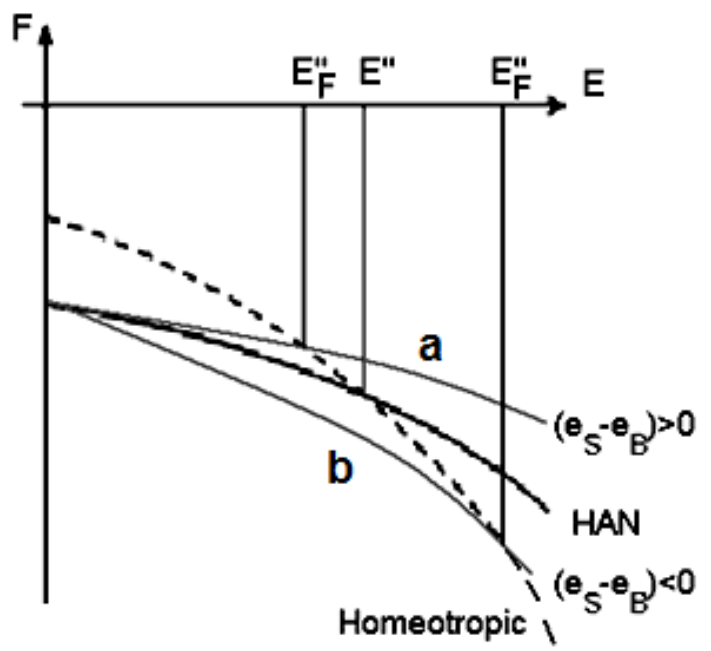

Figure 6: The two curves (a and b) show how the energy of HAN configuration changes for the presence of flexoelectricity. According the sign of the flexoelectric parameter, the threshold field is raised or lowered.

The thresholds change according to the shape of the molecules. Comparing the thresholds we can estimate the values of the coefficients. The two electric field contributions in the HAN cell are:

$$
\begin{aligned}
& F_{1}=-\frac{\varepsilon_{o} \Delta \varepsilon}{4} E^{2} S d \\
& F_{2}=-\left(e_{S}-e_{B}\right) E S
\end{aligned}
$$


If energies were of the same order of magnitude, we could obtain:

$$
\left(e_{S}-e_{B}\right) \approx \frac{\varepsilon_{o} \Delta \varepsilon}{4} E d
$$

In the case of a cell with a thickness of $10 \mu m$, a field of $10 \mathrm{~V} / \mu \mathrm{m}$, and an electric anisotropy as $\Delta \varepsilon=0.1$ we obtain:

$$
\left(e_{S}-e_{B}\right) \approx 25 \frac{p C}{m}
$$

in agreement with Ref.14 and with other experimental values [15,16]. Even a giant flexoelectricity has been found with bent-core nematics: a peak of $35 \mathrm{nC} / \mathrm{m}$ was measured in these materials then more than 3 orders of magnitude larger than in calamitics [17].

\section{Saddle-splay elasticity, PHAN cell and threshold thickness}

In nematics, a more general form of the distortion freeenergy density, in the framework of the usual first-order continuum theory, is given as:

$$
\begin{aligned}
& f=\frac{K}{2}\left\{(\operatorname{div} \vec{n})^{2}+(\vec{n} \cdot \operatorname{rot} \vec{n})^{2}+(\vec{n} \times \operatorname{rot} \vec{n})^{2}\right\} \\
& -\left(K+K_{24}\right) \operatorname{div}[\vec{n} \operatorname{div} \vec{n}+\vec{n} \times \operatorname{rot} \vec{n}]
\end{aligned}
$$

The last term in (28) is the contribution of the saddle-splay elasticity $[4,5]$. In fact, this contribution is not usually inserted in the bulk free energy, because it becomes a surface contribution when integration is made on the cell thickness. In addition to the anchoring energy then, there is an elastic contribution too.

Saddle-splay contributions are relevant in evaluating the elastic contribution of thin films or membranes $[12,18]$. Sometimes, periodic distortions of the director in the HAN cells are observed [4,5]. Because of this periodic configuration, the cell is in a PHAN configuration, that is a nematic cell with a period hybrid alignment. Two angles describe the PHAN configuration: $\theta$ and $\varphi$. The last angle is formed by the projection of the director in the plane of the cell with the $x$-axis. The frame of reference is $[x y z]$, with $[x y]$ the cell plane and the $z-$ axis perpendicular to the cell plane (Figure 7).

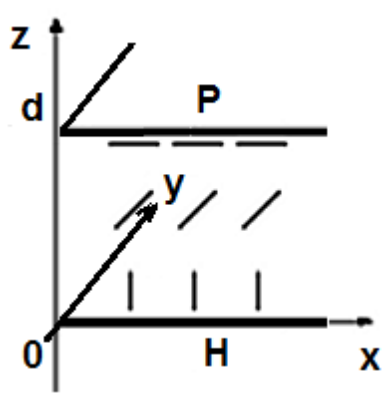

Figure 7: Frame of reference for the PHAN cell.

The homeotropic wall is at $z_{0}=0$, where $z$ is the axis perpendicular to the cell plane. The planar wall is at $z_{1}=d$, where $d$ is the thickness of the cell. The easyaxis of the planar alignment is chosen coincident with the $x$-axis. The director $\vec{n}$ is described as:

$\vec{n}=\vec{i} \cos \varphi \cos \theta+\vec{j} \sin \varphi \cos \theta+\vec{k} \operatorname{sen} \theta$

The Euler-Lagrange equations are non-linear. These equations were solved in Ref.4, with a numerical approach to determine the threshold thickness of the cell between the planar and the PHAN.

To grasp the role of the saddle-splay contribution, we can also use a simple calculation. Let us consider the tilt angle $\theta$ depending on $z$, and the $\varphi$ angle depending on $x$, in the following way:

$$
\theta(z)=\frac{\pi z}{2 d} \quad ; \quad \varphi(x)=\frac{2 \pi x}{\Lambda}
$$

The tilt is zero if $z=0$, and it is $\pi / 2$ at $z=d$. With $\Lambda$ we denote the wavelength along $x$-axis. The free energy density is:

$$
\begin{aligned}
f= & \frac{K}{2}\left\{\left(\frac{\pi}{2 d}\right)^{2}+\left(\frac{2 \pi}{\Lambda}\right)^{2} \sin ^{2} \varphi\right. \\
& \left.-\frac{2 \pi^{2}}{\Lambda d} \cos ^{2} \theta \sin \varphi\right\}
\end{aligned}
$$

Let us have a volume $V=d \Lambda D$, where $D$ is a fixed distance on $y$ - axis. We have:

$$
F=\frac{K}{2} \Lambda D d\left[\left(\frac{\pi}{2 d}\right)^{2}+\frac{1}{2}\left(\frac{2 \pi}{\Lambda}\right)^{2}\right]
$$


Neglecting the anchoring with respect to $\varphi$, and assuming just tilt anchoring, with a surface energy density of the form:

$f_{\text {Surf }}=-W n_{z}^{2}$

where $W=W_{P}$ for planar anchoring with $\theta=0$, and $W=W_{H}$ at the homeotropic anchoring $\theta=\pi / 2$. After integrating on surfaces of the cell:

$$
F_{\text {Surf }}=-\left(W_{P}+W_{H}\right) \Lambda D
$$

and then the total free energy is:

$$
\begin{gathered}
F=\frac{K}{2} \Lambda D d\left[\left(\frac{\pi}{2 d}\right)^{2}+\frac{1}{2}\left(\frac{2 \pi}{\Lambda}\right)^{2}\right] \\
-\left(W_{P}+W_{H}\right) \Lambda D
\end{gathered}
$$

Let us evaluate the saddle-splay contribution to free energy density, using Equation 4 of Ref.4. In the present approximation, it is:

$$
\begin{gathered}
f_{\text {Saddle-splay }}=2\left(1+\kappa_{4}\right) K\left[-\theta_{H}\left(\frac{\partial \varphi}{\partial x}\right)_{H}\right] \\
=2\left(1+\kappa_{4}\right) K\left[-\frac{\pi}{2} \frac{2 \pi}{\Lambda}\right]
\end{gathered}
$$

where $\kappa_{4}=K_{24} / K$; after integration on a surface $S=\Lambda D$, we have:

$$
F_{\text {Saddle-splay }}=-2\left(1+\kappa_{4}\right) K \pi^{2} D
$$

Adding (37) to (35), the total energy is:

$$
\begin{gathered}
F_{P H A N}=\frac{K \pi^{2} D \Lambda}{8 d}+\frac{K \pi^{2} d D}{\Lambda} \\
-\left(W_{P}+W_{H}\right) \Lambda D-2\left(1+\kappa_{4}\right) K \pi^{2} D
\end{gathered}
$$

Comparing with the free energy of HAN configuration:

$$
F_{P H A N} \approx F_{H A N},
$$

after simple calculations we find:

$$
d^{2}-2\left(1+\kappa_{4}\right) \Lambda d-\frac{1}{8} \Lambda^{2}=0
$$

The threshold value for the cell thickness:

$$
d_{c} \cong 4 \Lambda\left(1+\kappa_{4}\right)
$$

Therefore, if $d>d_{c}$, we find a HAN configuration, if $d<d_{c}$ the modulated PHAN texture is displayed in the cell. In Ref.4, we can see the experimental observation of thickness threshold in a nematic sample.

On the role of flexoelectricity and saddle-splay elasticity in creating the periodic distortion of the nematic director, we have made a discussion which is considering some approximations. Of course, more refined calculations are possible, but the present discussion is enough to understand the origin of thresholds for electric field and cell thickness in the nematic cells.

\section{References}

1. L.D. Landau and E.M. Lifshitz, Statistical physics, Pergamon Press, Oxford, UK, 1980.

2. P. Oswald and P. Pieranski, Nematic and cholesteric liquid crystals: Concepts and physical properties illustrated by experiments, Taylor \& Francis, 2005.

3. S.A. Pikin, Structural transformations in liquid crystals, Gordon \& Breach Science Publishers Ltd., 1991

4. A. Sparavigna, O.D. Lavrentovich and A. Strigazzi, Periodic stripe domains and hybrid-alignment regime in nematic liquid crystals: Threshold analysis. Phys. Rev. E 1994, 49, 1344-1352.

5. A. Sparavigna, L. Komitov, O. Lavrentovich and A. Strigazzi, Saddle-splay and periodic instability in a hybrid aligned nematic layer subjected to a normal magnetic field. Journal de Physique II, EDP Sciences 1992, 2 (10), 1881-1888.

6. L.K. Vistin, Electrostructural effect and optical properties of a certain class of liquid crystals and their binary mixtures, Sov. Phys. Crystallogr. 1970, 15(3) 514-515.

7. Y.P. Bobylev, V.G. Chigrinov and S.A. Pikin, Threshold flexoelectric effect in nematic liquid crystals, Journal de Physique Colloques 1979, 40(C3), 331-333.

8. O.D. Lavreontovich and V.M. Pergamenshchik, Periodic domain-structures in thin hybrid nematic layers, Mol. Cryst. Liquid Cryst. 1990, 179, 125-132.

9. L.D. Landau and E.M. Lifsits, Fisica Teorica 8 - Elettrodinamica dei mezzi continui, Editori Riuniti, Roma, 1986.

10. P.G. De Gennes and J. Prost, The Physics of Liquid Crystals, Oxford Science Publication, 1995

11. A.C. Sparavigna, Distortional Lifshitz vectors and helicity in nematic free energy density, International Journal of Sciences, 2013, 2(7), 54-59.

12. A. Sparavigna, Role of Lifshitz invariants in liquid crystals, Materials 2009, 2, 674-698.

13. R.B. Meyer, Piezoelectric effects in liquid crystals. Phys. Rev. Lett. 1969, 22, 918-921.

14. D.L.G. Cheung, Structures and properties of liquid crystals and related molecules from computer simulation, $\mathrm{Ph}$. D. Thesis, University of Durham, Durham, UK, 2002.

15. D. Schmidt, M. Schadt and W.Z. Helfrich, Liquid-crystalline curvature electricity: The bending mode of MBBA. Z. Naturforschung, 1972, 27a, 277-280.

16. S. Warrier and N.V. Madhusudana, An AC electrooptic technique for measuring the flexoelectric coefficient and anchoring energies of nematics, J. Phys. II 1997, 7, 1789-1803.

17. J. Harden, B. Mbanga, N. Ėber, K. Fodor-Csorba, S. Sprunt, J.T. Gleeson and A. Jákli, Giant flexoelectricity of bent-core nematic liquid crystals, Phys. Rev. Lett. 2006, 97, 157802-1-157802-4.

18. W.Z. Helfrich, Elastic properties of lipid bilayers: theory and possible experiments, Z. Naturforschung, 1973, 28c, 693-703. 